\title{
Relação entre vitamina $D$ e distúrbios metabólicos em mulheres menopausadas
}

\author{
Relationship between vitamin $d$ and metabolic disorders in menopausal women
}

Relación entre la vitamina D y los trastornos metabólicos en mujeres menopáusicas

Amanda Mendes Spirlandeli ${ }^{1}$, Luana Mara Maciel Machado Pulicano ${ }^{1 *}$, Fernanda Felipe ${ }^{1}$, Elisabete Lilian Dair ${ }^{1}$, Marcelo Ballaben Carloni'.

\section{RESUMO}

Objetivo: O trabalho objetiva estabelecer uma relação direta entre o perfil metabólico de mulheres menopausadas com a concentração sérica de vitamina D. Métodos: Foi realizado um estudo prospectivo com a participação de 25 mulheres na menopausa, em consulta no Ambulatório Escola universitário. Foi solicitado a elas exames de glicemia, colesterol total, HDL e LDL colesterol, triglicérides e vitamina D, além de avaliação de altura, peso, cálculo do Índice de Massa Corporal (IMC) e questionário sobre prática de atividade física, utilizando a correlação linear de Pearson para análise. Resultados: Observou-se relação inversa entre os valores de colesterol total e LDL, diferente do que foi observado em relação ao IMC, circunferência abdominal, HDL, em que houve fraca correlação. Sobre os valores de glicemia e da densitometria óssea, houve relação direta, porém de fraca magnitude. Conclusão: Dessa forma, o trabalho se equipara em alguns aspectos a outros já realizados sobre o assunto, sendo de grande importância a realização de novos estudos, uma vez que se for provada, a reposição de vitamina $D$ pode ser benéfica para minimizar risco cardiovasculares em menopausadas, como na população em geral.

Palavras chaves: Glicemia, Menopausa, Vitamina D.

\begin{abstract}
Objective: The study aims to establish a direct relationship between the metabolic profile of menopausal women with the serum concentration of vitamin D. Methods: A prospective study was carried out with the participation of 25 women in menopause, in consultation at the School Clinic of the University of Franca UNIFRAN. They were asked to perform tests for blood glucose, total cholesterol, HDL and LDL cholesterol, triglycerides and vitamin $\mathrm{D}$, in addition to assessment of height, weight, calculation of the Body Mass Index (BMI) and questionnaire on the practice of physical activity, using Pearson's linear correlation for analyze. Results: An inverse relationship was observed between the values of total cholesterol and LDL, different from what was observed in relation to BMI, waist circumference, HDL, in which there was a weak correlation. Regarding the blood glucose and bone densitometry values, there was a direct relationship, however of low magnitude. Conclusion: The work is in some ways similar to others already carried out on the subject, and it is of great importance to carry out new studies, since if proven, vitamin D replacement can be beneficial to minimize cardiovascular risk in menopause, as well as in the general population.
\end{abstract}

Keywords: Blood glucose, Menopause, Vitamin D.

\section{RESUMEN}

Objetivo: El estudio tiene como objetivo establecer una relación directa entre el perfil metabólico de la mujer menopáusica con la concentración sérica de vitamina D. Métodos: Se realizó un estudio prospectivo con la participación de 25 mujeres en menopausia, en consulta en la Clínica Escolar de la Universidad de Franca UNIFRAN. Se les solicitó realizar pruebas de glucemia, colesterol total, colesterol HDL y LDL, triglicéridos y vitamina D, además de valoración de talla, peso, cálculo del Índice de Masa Corporal (IMC) y cuestionario sobre la práctica de actividad física, utilizando el método lineal de Pearson. correlación para analizar. Resultados: Relación inversa entre los valores de colesterol total y LDL, diferente a lo observado en relación al IMC, circunferencia de cintura, HDL, en el que hubo una correlación débil. En cuanto a los valores de glucosa en sangre y densitometría ósea, hubo una relación directa, sin embargo, de débil magnitud. Conclusión: El trabajo es en cierto modo similar a otros ya realizados sobre el tema, y es de gran importancia realizar nuevos estudios, ya que, de comprobarse, el reemplazo de vitamina D puede ser beneficioso para minimizar el riesgo cardiovascular en menopausia, así como en la población en general.

Palabras clave: Glucemia, Menopausia, Vitamina D.

${ }^{1}$ Universidade de Franca (UNIFRAN), Franca - SP. *E-mail: luanamara1@hotmail.com

Programa Institucional de Bolsas de Iniciação Cientifica (PIBIC) UNIFRAN

SUBMETIDO EM: 3/2021

ACEITO EM: 4/2021

PUBLICADO EM: 4/2021 


\section{INTRODUÇÃO}

A vitamina $D$ é uma substância fundamental em diversas atividades do organismo, atuando na formação óssea como também na estrutura do colesterol e na secreção de insulina. Sua forma de aquisição pode ser a partir da alimentação, com ingestão de alguns óleos e peixes gordurosos, sendo que sua principal fonte de absorção se encontra na conversão de 7-de-hidrocolesterol (7-DHC) em pré-colecalciferol pelos raios ultravioleta B (UVB) (MAEDA SS, et al., 2014).

Os valores de vitamina $D$ estabelecidos pela Sociedade Brasileira de Patologia Clínica/Medicina Laboratorial (SBPC/ML) e Sociedade Brasileira de Endocrinologia e Metabologia (SBEM), para uma população saudável é maior ou igual a $20 \mathrm{ng} / \mathrm{mL}$. Recomenda-se números entre 30 e $60 \mathrm{ng} / \mathrm{mL}$ para idosos, gestantes e para pacientes com doenças autoimunes e metabólicas, como osteoporose. Sendo, portanto a hipovitaminose definida com valores entre 10 e $20 \mathrm{ng} / \mathrm{mL}$, enquanto em números menores do que $10 \mathrm{ng} / \mathrm{mL}$, observa-se o risco de desenvolvimento de osteomalácia, e raquitismo (SBEM, 2019).

Embora o papel no metabolismo ósseo seja o mais reconhecido, atualmente vários estudos vêm demonstrando inúmeras outras funções oriundas da vitamina $D$. Estima-se que tal componente atue em cerca de $3 \%$ das atividades do corpo humano, controlando funções fundamentais ao equilíbrio sistêmico, bem como durante a apoptose celular, no sistema cardiovascular, metabolismo lipídico e em atividades endocrinológicas (CASTRO LCGD, 2011).

As principais manifestações causadas pela diminuição da vitamina $D$ podem ser divididas em dois tipos: ósseas, como osteopenia e osteoporose. Situação frequente com o avanço da idade e, sendo mais comum em mulheres na pós-menopausa, possuindo como etiologia fatores genético, ambientais e até mesmo geográficos (KHASHAYAR P, et al., 2016).

Apesar da anterior ser mais conhecida, estudiosos começaram a perceber a possibilidade de interferência dessa vitamina com o aumento ou diminuição da prevalência de diabetes melitus e colesterol LDL e HDL. Assim a deficiência em tal substância passou a ser alvo de uma associação com o desenvolvimento da Síndrome Metabólica (SM) (SIMAS LAW, et al., 2019).

Essa condição pode ser definida com a presença de pelo menos 3 ou mais dos seguintes critérios: intolerância à glicose com glicemia de jejum $\geq 100 \mathrm{mg} / \mathrm{dl}$; obesidade abdominal ou maior quantidade de gordura visceral com circunferência da cintura $>90 \mathrm{~cm}$ para homens e $>80 \mathrm{~cm}$ para mulheres; triglicerídeos $\geq 150 \mathrm{mg} / \mathrm{dl}$; lipoproteína de alta densidade (HDL) colesterol < $40 \mathrm{mg} / \mathrm{dl}$ para homens e $50 \mathrm{mg} / \mathrm{dl}$ para mulheres; Terapia anti-hipertensiva vigente ou pressão $\geq 130 \times 85$ mmHg (FONSECA EJNDC, et al, 2018).

A partir de pesquisas, foi possível demonstrar o papel fisiopatológico e bioquímico da vitamina $D$ nos distúrbios endocrinometabólico. No entanto, falta evidencias a respeito do seu papel como terapêutica e suplementação, uma vez que ensaios clínicos realizados com sua adição, não se mostraram capazes de minimizar as chances de desenvolvimento de doenças metabólicas (JORGE AJL, et al., 2018).

Por ter forte relação à sua função ligada a luz solar, tem seus valores intimamente relacionados com quantidade e duração da incidência de sol no globo, sendo menor em regiões mais próximas dos polos, bem como durante os meses de inverno. Outra população que sofre com a baixa de vitamina $D$ são os idosos, os quais muitas vezes necessitam de reposições dessa vitamina para regulação da homeostase corporal (GALVÃO LO, et al., 2013).

Outro item importante que se relaciona com a vitamina $D$ seria a prática de atividade de física, a qual é definida pela Organização Mundial da Saúde (OMS) como movimento do corpo que feito pelos músculos esqueléticos que resultem em gasto energético como atividade doméstica, viagens, lazer. Tal prática promove o aumento da concentração do nível de HDL e redução significativa dos triglicerídeos. Contudo, sua influência sobre a concentração de LDL é menos notória (SBC, 2017).

Ela também influencia positivamente no metabolismo da glicose, diminuindo a secreção de insulina, e aumento do transportador de glicose 4 (GLUT4), com aumento da capacidade de transportar glicose para o interior da célula, melhorando a sensibilidade periférica a insulina. Os exercícios resistidos tem maior eficácia em comparação ao aeróbio devido a capacidade de armazenamento da glicose na célula muscular (SBD, 2017; SBD, 2018). 
Dessa forma, percebemos como sedentarismo pode ser fator influenciador nesse processo. Assim, de acordo com a Associação Brasileira para o Estudo da Obesidade e síndrome metabólica (ABESO), sedentarismo é definido como qualquer sujeito que não desempenha atividade física regular em grande parte das semanas (ABESO, 2016).

Em vista da relação com as funções endocrinometabólicas, um grupo importante a ser estudado são menopausadas. Período correspondente a última menstruação feminina, definida por amenorreia por mais 12 meses (FEBRASGO, 2010). Fase que cursa com redução da produção de estrogênio, que auxiliava proteção da mulher contra eventos cardiovasculares, associado ao fator idade, favorecendo naturalmente a redução da vitamina $D$, torna-se esse grupo alvo para estudos sobre benefícios do uso exógeno de 25(OH) (SIMAS LAW, et al., 2019).

Nessas mulheres, além da diminuição dos níveis de estrogênio, associado a maior susceptibilidade hipovitaminose $D$, soma-se também a maiores perdas de massa magra em oposição ao aumento de massa gordurosa (SIMAS LAW, et al., 2019). Dessa forma, elas se tornam grande alvo para desenvolvimento de riscos cardiovasculares e consequentemente a SM, uma vez que uma média de $50 \%$ desse grupo com mais de 50 anos são diagnosticadas com hipovitaminose D, acarretando grande valor em morbimortalidade (SCHMITT EB, et al., 2017).

Além da redução da vitamina $D$ em mulheres pós-menopausa, pode-se observar, muitas vezes, um aumento indiretamente proporcional do hormônio PTH (GALVÃO LO, et al., 2013). Evidencias mostram que tal hormônio, tem papel responsável na lipólise, sendo, portanto suprimido na presença de níveis elevados ou normais de $25(\mathrm{OH}) \mathrm{D}$. Com isso, a partir da diminuição da vitamina, ocorre uma quebra no eixo de regulação, gerando uma balanço negativo no perfil lipídico (MILANI P, 2014).

Em vista da forte relação positiva da vitamina $D$, foi verificado que após reposição em mulheres pósmenopausadas com doenças já estabelecidas, como DM2, obesidade, HAS, não foi observado melhora significativa no desenvolvimento da patologia. O que evidencia a necessidade de maiores estudos sobre 0 tema. Além disso, fica o questionamento acerca da função da vitamina $D$ como profilaxia de doenças cardiovasculares e SM e, não como tratamento (JORGE AJL, et al., 2018).

Devido a não concordância na literatura sobre os benefícios, a investigação entre relação da vitamina $D$ com o perfil lipídico e a resistência insulínica é necessária, uma vez que esses indicadores se correlacionam com o desenvolvimento de doença cardiovascular. Com relação estabelecida, benefícios serão gerados ao grupo estudado, levando a pesquisas e tratamentos. Assim, tem-se o objetivo de estabelecer relação na concentração sérica da vitamina $\mathrm{D}$ com os níveis de colesterol, glicemia, IMC, atividade física e circunferência abdominal (CA) com os distúrbios metabólicos.

\section{MÉTODOS}

O projeto foi aprovado pelo comitê de ética em Pesquisa da Santa Casa de Misericórdia de Franca sob o Número do Parecer: 4.267.745 e Número do CAAE Número do CAAE: 24335619.0.3001.5438. Elaborado partir de uma análise bibliográfica entre plataformas digitais, foi buscado temas como perfil lipídico, CA, atividade física, glicemia, IMC, menopausa, vitamina $D$.

$\mathrm{O}$ artigo constou de 25 participantes, regularmente agendadas em consulta de Ginecologia em um Ambulatório Escola Universitário. O início da coleta de dados teve seu processo atrasado pela presença da pandemia de SARS COV-2/COVID 19 iniciada em Março/2020, cujo impacto resultou no fechamento do Ambulatório, adiando a pesquisa, aliado a demora já esperada do resultado de alguns exames, como densitometria óssea, além da própria disponibilidade da participante em realizar os exames solicitados.

Coletou-se dados de circunferência abdominal, índice de massa corporal e questionário de atividade física no próprio ambulatório escola, e solicitado à participante a realização dos exames laboratoriais e imagem em serviços conveniados pelo Sistema Único de Saúde (SUS). Além desses itens, foi questionado a participante sobre uso de medicações diárias, uma vez que podem influenciar nos resultados dos exames solicitados.

As participantes que aceitaram participar da pesquisa livremente, receberam informações a respeito dos riscos e benefícios da pesquisa, bem como explicação de que os dados obtidos seriam para fins de estudo e que suas identidades não seriam violadas. Após isso, foi entregue a elas o Termo de Consentimento Livre e Esclarecido (TCLE), em que após a leitura, elas assinaram-no aceitando sua participação. 
Os exames laboratoriais foram: glicemia de jejum, perfil lipídico (LDL-colesterol, HDL- colesterol, Triglicérides e colesterol total), vitamina D. Cuja referência para glicemia acima de $100 \mathrm{mg} / \mathrm{dl}$ se enquadra em um perfil de risco para desenvolvimento de diabetes mellitus. Para LDL foi considerado valores $>160 \mathrm{mg} / \mathrm{dll}$, HDL para mulheres menor ou igual a $50 \mathrm{mg} / \mathrm{dl}$, triglicérides valores $>150 \mathrm{mg} / \mathrm{dl}$ e colesterol total desejável < $200 \mathrm{mg} / \mathrm{dl}$, assim sendo, valores fora do esperado foram considerados alterados.

Em relação a densitometria óssea (DMO), analisando as variáveis em T-escore em L1-L4 e colo, que compara níveis de densidade óssea de pessoas de mesmo sexo e idade. Os valores considerados normais são T-score $\geq-1$, se entre -1 e -2,5 representa baixa massa óssea e $<-2,5$ demonstra osteoporose.

O IMC feito a partir da avaliação da altura/metro ${ }^{2}$ em balança antropométrica mecânica, e considerou-se baixo peso $<18,5$, peso ideal entre 18,6-24,9, sobrepeso 25-29,9 e obesidade grau I 30 a 34,9, obesidade grau II 35-39,0 e obesidade grau III >40. CA foi feita a partir da medição com uma fita métrica do ponto médio entre a crista ilíaca e o rebordo costal inferior. Ambos os procedimentos foram realizados durante 0 atendimento da participante no ambulatório escola.

Sobre sedentarismo e atividade física foi realizado o Questionário Global de Atividade Física (GPAQ) modificado no próprio ambulatório escola, englobado apenas o nível de atividade de média e elevada intensidade no seu tempo livre, levando em consideração o número de dias e minutos dedicados a pratica esportiva (Tabela 4).

Os valores dos exames foram dispostos na tabela a seguir, em que na sequência realizou-se análise estatística a fim de relacionar os valores dos exames com a vitamina $D$. Para tal, foi utilizado a correlação linear de Pearson, em que o coeficiente de correlação $(r)$ representa medida de associação linear com as variáveis. O valor $r$ varia de -1 a 1, e o sinal negativo ou positiva apenas indica a direção da relação, enquanto o valor obtido indica a força dessa associação. Uma correlação perfeita (-1 ou 1) indica que o escore de uma variável pode ser determinado exatamente ao se saber o escore da outra (FILHO DBF e JÚNIOR JADS, 2009) (Tabela 1).

Tabela 1 - Valores obtidos dos exames laboratoriais e dados de exame físico das participantes.

\begin{tabular}{|c|c|c|c|c|c|c|c|c|c|c|}
\hline & \multirow[t]{2}{*}{ Glicemia } & \multirow[t]{2}{*}{ LDL } & \multirow[t]{2}{*}{ HDL } & \multirow[t]{2}{*}{ TG } & \multirow[t]{2}{*}{ Vitamina D } & \multirow[t]{2}{*}{$\begin{array}{c}\text { Colesterol } \\
\text { total }\end{array}$} & \multirow[t]{2}{*}{ IMC } & \multirow[t]{2}{*}{ CA } & \multicolumn{2}{|c|}{$\begin{array}{c}\text { Densitometria } \\
\text { Óssea }\end{array}$} \\
\hline & & & & & & & & & L1-L4 & Colo \\
\hline 1 & 104 & 174 & 55 & 151 & 21,6 & 259 & 22,9 & 70 & $-0,5$ & $-1,8$ \\
\hline 2 & 113 & 163 & 53 & 98 & 16,9 & 237 & 23,5 & 72 & 2,4 & $-1,2$ \\
\hline 3 & 106 & 108 & 46 & 199 & 9,8 & 186 & 24,1 & 82 & $-0,7$ & $-2,2$ \\
\hline 4 & 168 & 171 & 42 & 255 & 16,6 & 255 & 24,46 & 94 & $-0,4$ & -2 \\
\hline 5 & 98 & 69 & 41 & 59 & 25,4 & 124 & 26,2 & 96 & $-0,9$ & $-0,1$ \\
\hline 6 & 100 & 89 & 75 & 142 & 35,8 & 161 & 27,5 & 98 & $-0,8$ & -1 \\
\hline 7 & 103 & 84 & 48 & 117 & 31,7 & 153 & 28 & 98 & 1,8 & 0,7 \\
\hline 8 & 83 & 117 & 83 & 62 & 17,8 & 215 & 29,2 & 99 & $-1,6$ & 0,2 \\
\hline 9 & 100 & 155 & 73 & 126 & 24,3 & 253 & 30,5 & 101 & 0,7 & 0,2 \\
\hline 10 & 97 & 110 & 57 & 143 & 19,6 & 193 & 30,5 & 104 & $-1,6$ & $-1,8$ \\
\hline 11 & 94 & 185 & 68 & 154 & 21,2 & 283 & 31,2 & 105 & $-1,3$ & $-2,6$ \\
\hline 12 & 96 & 187 & 62 & 107 & 16,3 & 272 & 31,5 & 108 & $-2,1$ & 0,3 \\
\hline 13 & 96 & 75 & 52 & 123 & 46,6 & 147 & 33,2 & 109 & $-1,2$ & $-1,6$ \\
\hline 14 & 98 & 158 & 76 & 71 & 25,3 & 251 & 33,5 & 111 & $-0,9$ & $-1,1$ \\
\hline 15 & 93 & 111 & 75 & 180 & 20,8 & 216 & 35,9 & 120 & -1 & $-0,8$ \\
\hline 16 & 100 & 136 & 50 & 121 & 26,9 & 163 & 36 & 79 & $-1,5$ & $-0,2$ \\
\hline 17 & 101 & 178 & 59 & 333 & 16,7 & 293 & 37,6 & 77 & $-0,9$ & 0,2 \\
\hline 18 & 99 & 183 & 68 & 75 & 19,4 & 269 & 41,2 & 85 & 1,6 & 1 \\
\hline 19 & 98 & 123 & 47 & 73 & 18,8 & 187 & 30,4 & 102 & $-1,3$ & $-0,2$ \\
\hline 20 & 98 & 130 & 53 & 169 & 32,8 & 214 & 31 & 99 & 0,6 & $-1,5$ \\
\hline 21 & 103 & 134 & 67 & 150 & 27,3 & 229 & 30,8 & 98 & 0,8 & $-0,3$ \\
\hline 22 & 90 & 160 & 83 & 84 & 19,7 & 262 & 24,3 & 94 & $-0,1$ & $-2,7$ \\
\hline 23 & 89 & 96 & 65 & 73 & 23,7 & 177 & 24 & 93 & $-2,1$ & $-0,7$ \\
\hline 24 & 105 & 142 & 69 & 80 & 25,5 & 228 & 23 & 94 & 0,2 & $-0,7$ \\
\hline 25 & 85 & 109 & 86 & 53 & 19,1 & 209 & 22,9 & 104 & $-0,8$ & $-0,7$ \\
\hline
\end{tabular}

Fonte: Spirlandeli AM, et al., 2021. 


\section{RESULTADOS}

A partir dos valores obtidos sobre os parâmetros avaliados nas participantes, foi construído a tabela 2 , como dados analisados pela correlação linear de Pearson. $O$ valor de ' $r$ ' encontrado demonstra a associação dos valores de vitamina $D$ com cada uma das variáveis pesquisadas (Tabela 2).

Tabela 2 - Análise de variáveis em relação aos níveis de vitamina D obtidos em mulheres menopausadas.

\begin{tabular}{ccccc}
\hline Variáveis & $\begin{array}{c}\text { Valores } \\
\text { alterados (\%) }\end{array}$ & $\begin{array}{c}\text { Valores dentro da } \\
\text { normalidade (\%) }\end{array}$ & $\mathbf{r}$ & $\begin{array}{c}\text { VALOR DA } \\
\text { RELAÇÃO }\end{array}$ \\
\hline LDL & 36 & 64 & $-0,49107$ & MÉDIA \\
TG & 32 & 68 & $-0,1496$ & FRACA \\
HDL & 20 & 80 & $-0,0493$ & FRACA \\
IMC & 68 & 32 & 0,05903 & FRACA \\
$\begin{array}{c}\text { Glicemia } \\
\text { Circunferência }\end{array}$ & 44 & 56 & $-0,15332$ & FRACA \\
Abdominal & 84 & 16 & 0,290842 & FRACA \\
Colesterol Total & 64 & 36 & $-0,50384$ & FORTE \\
\hline & \multicolumn{4}{|}{} \\
\hline L1-L4 & 32 & Densitometria óssea & \\
Colo & 40 & 68 & 0,08002 & FRACA \\
\hline
\end{tabular}

Fonte: Spirlandeli AM, et al., 2021.

Além disso, de acordo com a Tabela 3, em que foi elaborado uma média e mediana dos valores de vitamina D encontrado nas participantes, $52 \%$ delas se encontravam com valor de vitamina $D$ em níveis adequados e que a média de valores encontrados foi de $23,184 \mathrm{ng} / \mathrm{mL}$.

Tabela 3 - Média e mediana dos valores obtidos de vitamina D das participantes.

\section{VALORES DE VITAMINA D DAS}

PARTICIPANTES

\begin{tabular}{cc}
\hline MÉdia & MEDIANA \\
23,184 & 20,25 \\
\hline
\end{tabular}

Fonte: Spirlandeli AM, et al., 2021.

Como referência temos os valores de $r$ : entre 0,10 e 0,29 fraca associação; valores entre 0,30 e 0,49 associação média; 0,50 e 1 forte associação. Além disso, é importante classificar que o valor $r=1$ demonstra uma correlação positiva, isto é, diretamente proporcional. Enquanto $r=-1$ demonstra uma relação inversamente proporcional. $O$ valor $r=0$ demonstra que as variáveis não possuem relação linear, podendo ter uma correlação de outra natureza.

De acordo com os valores estabelecidos para r, é possível observar que LDL-colesterol, triglicérides, glicemia e colesterol total possuem relação inversa aos valores de vitamina $\mathrm{D}$, isto é, quanto mais alto os valores dessas variáveis, menor foi o valor obtido de vitamina $D$ nas participantes, ou vice-versa. $O$ contrário é observado aos valores HDL-colesterol, IMC, CA e os valores obtidos na densitometria óssea, em que demonstraram relação positiva com as variações de vitamina $D$.

No entanto, dos elementos analisados, apenas LDL-colesterol e colesterol total possuem correlação forte com os valores de vitamina $\mathrm{D}$, demonstrando que há relação significativa, com aumento do perfil lipídico e a queda dos níveis de vitamina $D$, ou mesmo que em participantes com hipovitaminose são mais susceptíveis ao aumento dos níveis desses parâmetros. Todavia, os valores de HDL-colesterol e IMC possuem valores próximos de ZERO, o que pode significar pouca relação com os níveis de vitamina $D$.

Sobre os valores na DMO observa-se correlação positiva, porém de fraca magnitude. A positividade na correlação demonstrada na associação é uma situação já esperada, uma vez que a vitamina $D$ é um elemento importante na formação da densidade mineral óssea.

Levando em consideração as relações observadas na Tabela 3, valores de perfil lipídico foram colocados no Gráfico 1, afim de tornar a visualização mais prática. As linhas traçadas nos gráficos, com as respectivas numerações, demarcam o ponto limite das variáveis, de acordo com a SBC, obtidas das participantes. 
Desses valores, $36 \%$ das participantes estavam com LDL-colesterol acima do esperado (160mg/dl), $20 \%$ estavam com valor de HDL abaixo do ideal, $32 \%$ com o triglicérides elevado e $64 \%$ estavam com o colesterol total acima do esperado. Dessa forma, correlacionando com os dados anteriores, nota-se que cerca de 113 das participantes possuem o colesterol elevado, o qual representa uma das principais associações com baixo índice de vitamina D (Tabela 3).

Gráfico 1 - Valores do lipidograma das participantes.

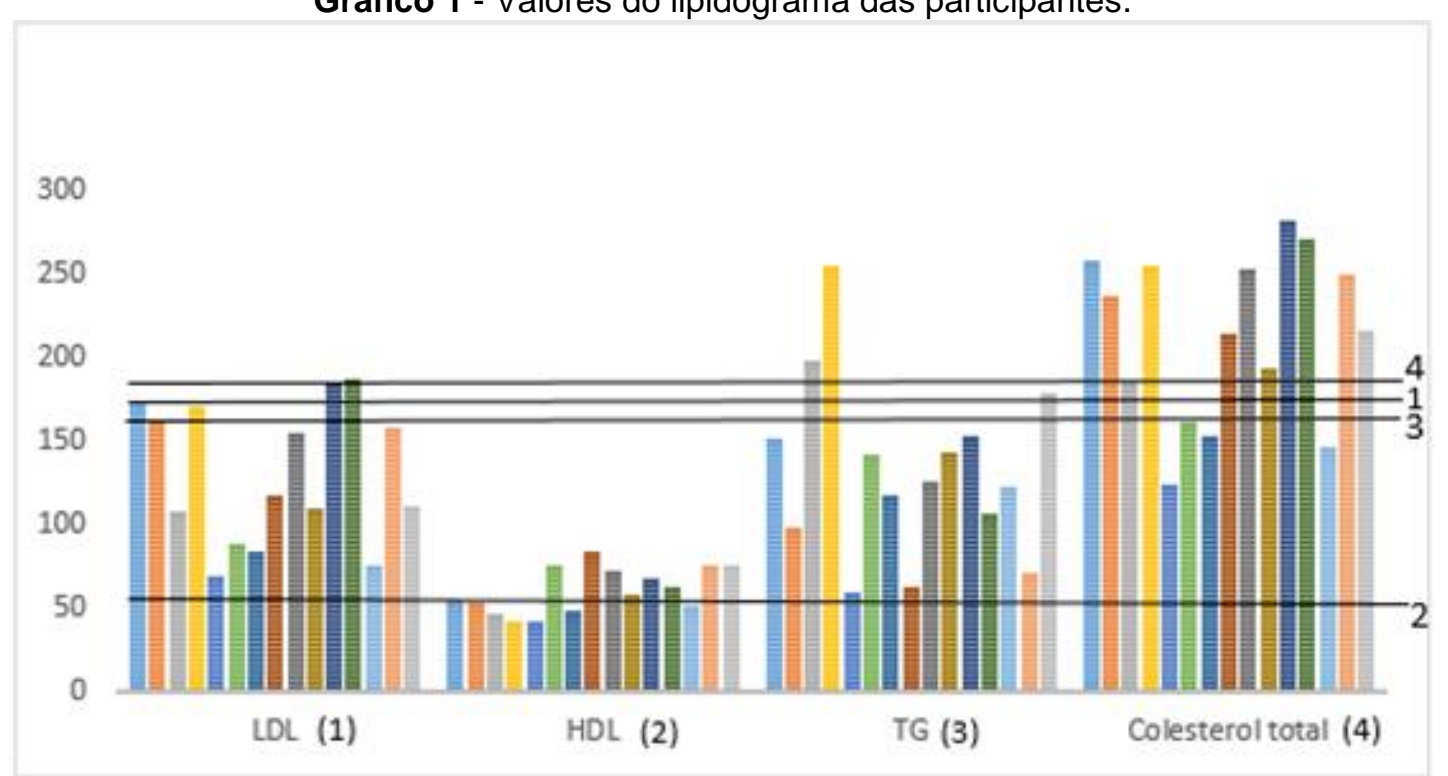

Fonte: Spirlandeli AM, et al., 2021.

Outro parâmetro é sobre medicações utilizadas pelas pacientes. Ao serem questionadas sobre elas, apenas uma paciente diz não fazer uso de nenhuma medicação, as outras referem uso de alguma medicação, e se dividem entre uso de algum tipo de antidepressivo e de fármacos anti-hipertensivos. Uma das participantes faz uso de alendronato de cálcio e nenhuma referiu fazer uso de medicação para tratamento de dislipidemia.

Sobre atividade física, a maioria das pacientes (90\%) são sedentárias, 6\% realizam atividades consideradas intensas e $11 \%$ realizam atividade de média intensidade, valores esses em relação as participantes ativas. As participantes que realizam atividades físicas possuem níveis de vitamina $D$ na faixa ideal para idade, bem como exemplificado pela média aritmética dos valores obtidos pelas mulheres e listados na Tabela 4 e 5.

Tabela 4 - Relação de participantes a respeito da prática de atividade física a partir de respostas obtidas pelo questionário GPAQ.

\begin{tabular}{|c|c|c|c|c|}
\hline \multicolumn{5}{|c|}{ Atividade Física Intensa } \\
\hline Realiza atividade física intensa & $\begin{array}{c}\text { SIM } \% \\
6 \%\end{array}$ & $\begin{array}{c}\text { NÃO \% } \\
94 \%\end{array}$ & & \\
\hline Frequência atividade & & & 5 dias* $^{*}$ & \\
\hline Duração da Atividade & & & & 40 minutos $^{\star *}$ \\
\hline
\end{tabular}

\begin{tabular}{|c|c|c|c|c|}
\hline \multicolumn{5}{|c|}{ Atividade Física Média Intensidade } \\
\hline $\begin{array}{l}\text { Atividades de intensidade média por pelo } \\
\text { menos } 10 \text { minutos }\end{array}$ & $\begin{array}{l}\text { SIM } \% \\
11,70 \%\end{array}$ & $\begin{array}{l}\text { NÃO\% } \\
88,23 \%\end{array}$ & & \\
\hline $\begin{array}{l}\text { Frequência que realiza atividades de } \\
\text { intensidade média }\end{array}$ & & & 5 dias* $^{*}$ & \\
\hline $\begin{array}{l}\text { Quanto tempo dedica a atividades de } \\
\text { nível médio }\end{array}$ & & & & 40 minutos $^{* *}$ \\
\hline
\end{tabular}


Tabela 5 - Media de pacientes que fazem atividade física e valores de vitamina D.

\begin{tabular}{cc} 
Média dos valores de Vitamina $D$ de & Média dos valores de Vitamina $D$ de \\
participantes que não fazem atividades física & participantes que fazem atividades física \\
\hline
\end{tabular}

21,98182

29,03333

Fonte: Spirlandeli AM, et al., 2021.

\section{DISCUSSÃO}

Incialmente é preciso conceituar a importância da avaliação da vitamina $D$ nessas mulheres, visto que no número amostral pequeno do trabalho, cerca de $40 \%$ das mulheres se encontravam com níveis abaixo de $20 \mathrm{ng} / \mathrm{mL}$ sendo um fator contribuinte para alteração da homeostasia do organismo. É sabido que a hipovitaminose $D$ é mais frequente em mulheres, com uma proporção de $66,7 \%$ em mulheres e $33,3 \%$ em homens. No Brasil, observou-se que a média de mulheres que se encontram nessa faixa é de cerca de $17 \%$ e que localidades em latitudes mais elevadas possuem níveis melhores do que mulheres vivendo ao sul do país (FEBRASGO, 2017)

Em relação a formação de colesterol, estudiosos demonstraram que a 25(OH) estaria relacionada direta e indiretamente a formação deste, tanto atuando sobre a formação de apopoproteina A, culminando na síntese de HDL, como sobre outros hormônios, como paratormônio e calcitriol, formadores de ácidos graxos e triglicérides (SOUZA WND, 2016).

No grupo amostral, notou-se que há forte relação na associação da vitamina D com os níveis de LDL e colesterol, sendo um dos parâmetros também vislumbrados em demais estudos. Como exemplo, têm-se uma pesquisa feita com 106 mulheres pós-menopausadas do Paraná, na qual se obteve correlação inversa entre os valores de vitamina $\mathrm{D}$ em relação aos níveis séricos de colesterol total, LDL- colesterol e triglicérides, o que reforça perfil lipídico desfavorável para mulheres com insuficiência de 25(OH) (SIMAS LAW et al., 2019). No entanto, nela obteve-se correlação negativa em relação a estrutura corporal, bem como foi possível analisar no presente trabalho, uma vez que a relação de IMC e circunferência corporal, não obteve relação satisfatória.

Em um estudo recente com 3434 participantes, em que houve suplementação de vitamina D para metade do grupo e para outro foi administrado placebo, notou-se efeito positivo na "redução do colesterol total sérico, colesterol LDL e níveis de triglicerídeos, mas não os níveis de colesterol HDL" no grupo em que foi empregado a suplementação (DIBABA DT, 2019).

Sobre aos valores de glicemia, observa-se também correlação inversa com os valores de vitamina D. Artigos publicados recentemente demonstram como vitamina $D$ influencia na gênese do diabetes mellitus (DM) tipo 1 e tipo 2, ao inibir produção de citocinas e interleucinas, além também de sensibilizar os receptores periféricos de insulina (MAEDA SS, et al., 2014).

Algumas pesquisas avaliaram o nível de vitamina D presente em indivíduos já com a DM 2 estabelecida, tendo como resultado hipovitaminose. Todavia, como é doença encontrada com mais frequência em idade avançada, que já contribui para redução de $25(\mathrm{OH})$, além de outros fatores que atuam como viés, como exposição solar, uso ou não de terapia hormonal, bem como tratamento para osteoporose.

Além disso, observa-se que grande parte das participantes se encontram com IMC de sobrepeso ou obesidade. Uma série de fatores podem influenciar para o surgimento dessa condição nas mulheres, como menor prática de exercícios, mudanças na alimentação e alterações inerentes do próprio envelhecimento (GONÇALVES JTT, et al, 2016). No entanto, a partir da análise estatística, nota-se que ambos os fatores citados se correlacionam fracamente com a vitamina $D$ na população estudada. $O$ que diferencia de outras pesquisas acerca do tema.

Paralelamente, estudos internacionais mostraram uma associação inversamente proporcional entre o valor de $25(\mathrm{OH})$ e obesidade. Mulheres com IMC $>34 \mathrm{~kg} / \mathrm{m}^{2}$ produzem cerca de $10 \%$ menos vitamina do que participantes do mesmo sexo com IMC próximo do adequado. A ainda não foi completamente elucidada, mas acreditam se tratar de uma união de fatores, como menor exposição solar por esse grupo, uma alteração do próprio metabolismo, em que o excesso de gordura corporal fosse o responsável por reter a vitamina $D$. (FORREST KYZ e STUHLDREHER WL, 2011). 
Outros demonstram que hipovitaminose $\mathrm{D}$ em obesos pode ser pelo aprisionamento desta nos adipócitos, culminando na diminuição da sua biodisponibilidade, reduzindo sua forma ativa na homeostase do organismo. Pesquisas relatam a importância da dosagem da vitamina nesse grupo, pois evidencias tem se mostrado promissoras com o uso de suplementação de $25(\mathrm{OH})$ na redução do peso (PERCEGONI N e CASTRO JMDA, 2014).

Em relação aos exercícios físicos, fica o questionamento a respeito da relação do aumento da $25(\mathrm{OH})$ sobre a pratica de atividades em ambiente fechado com os que praticam ao ar livre. No trabalho em questão, não foi aventado tal questionamento, sendo, portanto, um viés em relação ao resultado, contudo, com os resultados obtidos pelas participantes, nota-se que as que realizam exercícios físicos possuem valores de vitamina $D$ adequados para idade. Alguns estudos mostram a importância dessa relação enquanto outros relatam que os níveis de tal vitamina podem se basear a partir da modalidade de atividade, duração, intensidade e localização, e que exercícios mais intensos possuem uma associação relevante positiva mais forte com os valores de vitamina D (FERNANDES MR e JUNIOR WDRB, 2017).

A respeito das medicações em uso, torna-se difícil avaliar o real impacto que eles podem oferecer a pesquisa, uma vez que existe um viés nas respostas. Como as pacientes foram apenas questionadas sobre o uso de alguma droga, pode haver o risco do esquecimento por parte das pacientes. Mesmo sendo solicitado a lista de medicações em uso para ser trazido ao retorno, em alguns casos não foi possível o reencontro com a participante.

Por fim, a correlação entre os valores encontrados na densitometria óssea e vitamina $D$, evidenciam uma relação direta que é estabelecida por grandes estudos e já mais conhecida pelos profissionais na saúde em geral, porém no nosso grupo amostral, por ter um 'n' pequeno, o valor da relação foi baixo.

Tal situação já se tem evidências mais consistentes na literatura, sendo a dosagem de vitamina $D$ um componente importante na avaliação da estrutura óssea de mulheres na pós-menopausa, principalmente com osteoporose já reconhecida ou com fatores de risco, como também está em voga a sua dosagem na população geral. Sendo já recomendado, muitas vezes, sua suplementação isolada ou em associações com alendronato de cálcio.

\section{CONCLUSÃO}

Vitamina $D$ é um pró-hormônio fundamental nas atividades metabólicas do organismo, sendo adquirido pela alimentação ou pela radiação UVB convertendo 7-de-hidrocolesterol (7-DHC) em pré-colecalciferol. Estudos demonstram que a hipovitaminose $D$ seria um fator fundamental capaz de provocar alterações endócrinas no organismo, podendo ser influenciada pelo excesso de peso e sedentarismo. Assim sendo, o artigo evidencia que existe relação entre alguns parâmetros do perfil metabólico em menopausadas, porém se torna de fundamental importância a busca ativa de novos resultados, visto que se comprovada real correlação, os benefícios gerados pelo emprego de reposições da vitamina seriam expressivos e de baixo custo para população.

\section{AGRADECIMENTOS}

Agradecemos ao Programa Institucional de Bolsas de Iniciação Cientifica PIBIC/ UNIFRAN por fomentar a pesquisa, bem como agradecer a instituição por permitir utilizar sua infraestrutura na coleta de dados. E agradecer também a Fundação Santa Casa de Misericórdia de Franca por apoiar o projeto e acreditar que a pesquisa é fonte importante para avanços na saúde.

\section{REFERÊNCIAS}

1. ABESO. Associação Brasileira para o Estudo da Obesidade e da Síndrome Metabólica. Diretrizes Brasileiras de Obesidade. São Paulo, 2016; 4: 75.

2. CASTRO LCGD. O sistema endocrinológico vitamina D. Arquivos Brasileiros de Endocrinologia e Metabologia, 2011; 55(8): 566-75.

3. DIBABA DT. Effect of vitamin D supplementation on serum lipid profiles: a systematic review and meta-analysis. Nutrition Reviews, 2019; 77(12): 890-902.

4. FEBRASGO. Climatério. Manual de Orientação da Federação Brasileira das Associações de Ginecologia e Obstetrícia, São Paulo, 2010: 7-189. 
5. FEBRASGO. Federação Brasileira das Associações de Ginecologia e Obstetrícia. A importância da vitamina D na saúde da mulher. 2017; 14.

6. FERNANDES MR, JUNIOR WDRB. Association between physical activity and vitamin D: A narrative literature review. Revista Associação Medica Brasileira, São Paulo, 2017: 63(6): 550-556

7. FILHO DBF, JÚNIOR JADS. Desvendando os mistérios do coeficiente de correlação de pearson. Revista Política Hoje - UFPE, 2009; 18 (1): 115-146.

8. FONSECA EJNDC, et al. Síndrome metabólica e resistência insulínica pelo Homa-IR no climatério. International Journal of Cardiovascular Sciences, 2018; 31(3): 201-2018.

9. FORREST KYZ, STUHLDREHER WL. Prevalence and correlates of vitamin D deficiency in US adults. Nutrition Research, 2011; 31:48-54.

10. FRAGA AS, et al. Efeito do exercício sobre os níveis de HDL-c: uma revisão sistemática de metanálises. Revista Brasileira de Medicina do Esporte, 2017: 23(6): 488-494.

11. GALVÃO LO, et al. Considerações atuais sobre a vitamina D. Brasília Médica, 2013: 50(4): 324 - 332.

12. GOBBI B, et al. Avaliação de vitamina D por estação do ano em adultos de uma cidade no Sul do Brasil. Revista Brasileira de Analises Clinicas, 2016; 48(4): 341-345.

13. GONÇALVES JTT et al. Sobrepeso e obesidade e fatores associados ao climatério. Ciência \& Saúde Coletiva, 2016; 21(4): 1145-1155.

14. HYPPÖNEN E, BOUCHER BJ. Vitamin D, Obesity, and the Metabolic Syndrome. In: FELDMAN, D. Vitamin D. $4^{\text {th }}$ ed: Elselvier, 2018; 425p.

15. JORGE AJL, et al. Deficiência da vitamina d e doenças cardiovasculares. International Journal of Cardiovascular Science, 2018; 31(4): 422-432.

16. KHASHAYAR $P$, et al. Correlação entre os níveis de vitamina $D$ e densidade mineral óssea em uma população iraniana saudável. Revista Brasileira de Ortopedia, 2016; 51 (4): 454-458.

17. LICHTENSTEIN A, et al. A vitamina D: ações extraosseas e uso racional. Revista Associação Medica Brasileira, 2013; 59(5): 495-506.

18. MACHADO MRM. Prevalência da deficência de vitamina $D$ em mulheres na pós-menopausa no diagnóstico de câncer de mama. Dissertação (mestrado do curso de medicina). UNIVERSIDADE ESTADUAL PAULISTA (UNESP), Botucatu, 2018; $16 p$

19. MACIEL DG, REIS MJDA. Frequência de hipovitaminose D em mulheres adultas. Journal of the Health Sciences Institute, 2017; 35(4): 257- 260.

20. MAEDA SS, et al. Recomendações da Sociedade Brasileira de Endocrinologia e Metabologia (SBEM) para o diagnóstico e tratamento da hipovitaminose D. Arquivos Brasileiros de Endocrinologia e Metabologia, 2014: 58(5): 411433.

21. MILANI P. Correlação da vitamina D com o perfil lipídico e glicídico em indivíduos com síndrome metabólica. Dissertação (bacharel em enfermagem) Universidade Federal da Fronteira Sul, Chapecó: 2014.

22. MONTERA VDSP, MESQUITA ET. O papel da vitamina D na insuficiência cardíaca. Revista Brasileira de Cardiologia, 2010; 23(2): 124-130.

23. OMS. Atividade Física - nota informativa. Organização Mundial da Saúde - OMS, 2014; 385.

24. PERCEGONI N, CASTRO JMDA. Vitamina D, sobrepeso e obesidade - Uma revisão. HU Revista, 2014; 40(3, 4): 209-219.

25. PETERS BSE, MARTINI LA. Funções Plenamente Reconhecidas de Nutrientes Vitamina D. $2^{a}$ ed. São Paulo: ILSI Brasil, 2014.

26. PRÉCOMA DB, OLIVEIRA GMMD. Atualização da diretriz de prevenção cardiovascular da sociedade brasileira de cardiologia - 2019. Sociedade Brasileira de Cardiologia - SBC, 2019; 113(4): 787-891.

27. SBC. Sociedade Brasileira de Cardiologia. Atualização da diretriz Brasileira de dislipidemias e prevenção da aterosclerose, 2019.

28. SBD. Sociedade Brasileira de Diabetes. Diretrizes Sociedade brasileira de diabetes: Clannad, 2017-2018.

29. SBEM. Sociedade Brasileira de Endrocrinologia e Metabologia. Vitamina D: novos valores de referência. 2019. Disponível em: https://www.endocrino.org.br/vitamina-d-novos-valores-de-referencia/. Acesso em: 25 de Março de 2019.

30. SCHMITT EB, et al. Vitamin D deficiency is associated with metabolic syndrome in postmenopausal women. Maturitas, 2017; 107: 97-102.

31. SIMAS LAW, et al. Body composition and nutritional and metabolic parameters in postmenopausal women sufficient, insufficient and deficient in vitamin D. Archives of Endocrinology and Metabolism, 2019; 63(3): 265-271.

32. SOUZA WND. Concentrações séricas de 25(OH)D e perfil metabólico mediados pela adiposidade. Dissertação (Doutorado em Ciencias) - Faculdade de saúde pública. Universidade de São Paulo/USP, São Paulo, 2016.

33. WANG CM, et al. Inverse relationship between metabolic syndrome and 25-hydroxyvitamin D concentration in elderly people without vitamin D deficiency. Nature, 2018; 8(1): 17052.

34. WHO. Word Health Organization. Global Physical Activity Questionnaire GPAQ - Analysis Guide. WHO, 2012. 